\title{
Abundance and species diversity of predatory arthropods inhabiting rice of refuge habitats and synthetic insecticide application in freshwater swamps in South Sumatra, Indonesia
}

\author{
TILI KARENINA ${ }^{1}$, SITI HERLINDA ${ }^{2,3, \boldsymbol{v}}$, CHANDRA IRSAN ${ }^{2,3}$, YULIA PUJIASTUTI $^{2,3}$ \\ ${ }^{1}$ Program of Agriculture Sciences, Faculty of Agriculture, Universitas Sriwijaya. J1. Padang Selasa No. 524, Bukit Besar, Palembang 30139, South \\ Sumatra, Indonesia \\ ${ }^{2}$ Department of Plant Pests and Diseases, Faculty of Agriculture, Universitas Sriwijaya. Jl. Raya Palembang-Prabumulih, km 32, Indralaya, Ogan Ilir \\ 30662, South Sumatra, Indonesia. Tel.: +62-711-580663, Fax.: +62-711-580276, •email: sitiherlinda@ unsri.ac.id \\ ${ }^{3}$ Research Center for Sub-optimal Lands, Universitas Sriwijaya. Jl. Padang Selasa No. 524, Bukit Besar, Palembang 30139, South Sumatra, Indonesia
}

Manuscript received: 1 June 2019. Revision accepted: 28 July 2019.

\begin{abstract}
Karenina T, Herlinda S, Irsan C, Pujiastuti Y. 2019. Abundance and species diversity of predatory arthropods inhabiting rice of refuge habitats and synthetic insecticide application in freshwater swamps in South Sumatra, Indonesia. Biodiversitas 20: $2375-2387$. Rice cultivation in freshwater swamps in Indonesia is specific, among other things, there is no synthetic pesticide spraying and vegetable growing on rice field embankments. This specific cultivation technique can affect the abundance and diversity of rice-dwelling arthropods species. The study aimed to compare the abundance and species diversity of predatory arthropods inhabiting rice surrounded both by refugia and vegetables and by those being applied with synthetic insecticide in the rice field of freshwater swamps. This study used rice plots surrounded by refugia flower (Zinnia sp., Tagetes erecta, Cosmo caudatus, and Sesamum indicum); vegetables (Cucumis sativus, Vigna sinensis, Luffa acutangula, and Momordica charantia); untreated; and synthetic insecticide. Twenty-five herbivore species of 12 families, 34 spider species of 8 families, and 24 species of predatory insects of 12 families were found on rice during a planting season. The lowest herbivore population and spider abundance were found on synthetic insecticide sprayed rice and they were significantly different from those of refugia-rice plots. The most abundant spiders and predatory insects were found on rice surrounded by refugia and were not significantly different from those of rice surrounded by vegetables. The highest species diversity for spiders and predatory insects was found on rice surrounded by refugia, whereas the lowest species diversity was found on synthetic pesticide sprayed rice. Therefore, rice surrounded by refugia flowers and vegetables was the most appropriate for habitat and niche of predatory arthropods.
\end{abstract}

Keywords: Herbivore, neutral insect, parasitoid, predatory insect, spider

\section{INTRODUCTION}

Cultivation of rice (Oryza sativa) in freshwater swamps in Indonesia is specific, i.e., the planting season is from April to September in a year. Rice is planted by using transplanting method, i.e., starting from seedling and then transplanted to rice fields (Lakitan et al. 2018a,b). Rice seedling can be carried out with three patterns consisting of floating raft seedling, 'samir' method and digging ('tugal') method (Herlinda et al. 2018a; Lakitan et al. 2018b). Subsequently, seedlings of 7-10 days old are transplanted into rice fields. Rice pest control in freshwater swamp generally does not use synthetic insecticide (Herlinda et al. 2018b; Safitri et al. 2018), whereas weeds control is carried out without using herbicide but by using manual method through pulling out by hand or weeding by using a sickle. In addition, rice field embankments are usually planted with flowering seasonal vegetables, such as long beans (Vigna sinensis), cucumber (Cucumis sativus), bitter melon (Momordica charantia) and squash (Luffa acutangula).

This specific rice cultivation in freshwater swamps affects arthropod communities. Species richness and abundance of spiders on rice in freshwater swamps are higher than those of rice in tidal lowlands of South Sumatra because farmers in the freshwater swamps do not apply synthetic insecticide (Herlinda et al. 2018a). Application of synthetic insecticide can decrease the abundance and species diversity of predators and parasitoids (Ngin et al. 2017; Svobodová et al. 2017).

In addition to synthetic insecticide application, flora in the vicinity of rice can affect arthropod community (Cao et al. 2004; Lopes et al. 2017; Xu et al. 2017; Baba et al. 2018). Wild plants, refugia, and vegetables planted at rice field embankment can provide niche and habitat for arthropods as natural enemies of rice pest insects (Zhu et al. 2014a; Hasan et al. 2016; McCabe et al. 2017). Nectar and pollen on those crops and plants can increase longevity, fecundity, and function of natural enemies (Desai et al. 2017; Foti et al. 2017; Ganai et al. 2017; Lu et al. 2014). Some of refugia were utilized for natural enemy conservation on rice, for instance, Zinnia sp. becomes niche and habitat for spiders, Argiope aemula, Oxyopes sp., and Perenethis sp. (Desai et al. 2017). Tagetes erecta was inhabited by Oxyopes javanus, Coccinella septumpunctata, Syrphus spp., Geoceris spp., Apis dorsata, Apis mellifera, and Vanessa cardui (Ganai et al. 2017). Refugia species of Antigonon leptopus, Cassia cobanensis, Turnera subulata, and Antigonon leptopus were used for natural enemies' conservation of oil palm pests (Dutton et al. 2016; Kamarudin and Arshad 2016; Saleh and Siregar 2017). The 
effort to utilize refugia flower for predatory arthropod conservation in freshwater swamps of rice ecosystems in Indonesia has not yet been done. Therefore, this research aimed to compare the abundance and species diversity of predatory arthropods inhabiting rice of refuge habitats and synthetic insecticide applications in freshwater swamps.

\section{MATERIALS AND METHODS}

This research was conducted in the rice field of freshwater swamps in Pelabuhan Dalam Village, Pemulutan Subdistrict, Ogan Ilir District, South Sumatra Province, Indonesia from May to August 2018. The selected rice field is rice center, and its area is around 7.1 Mha that had flooding season from October to March and a dry season from April to September.

\section{Field preparation and treatments}

This stage consisted of land preparation and soil tillage, seedling, planting, and rice maintenance. Land preparation started with weeds clearance followed by soil tillage and application of manure fertilizer at a dose of 1 ton.ha ${ }^{-1}$. Rice seeds used in this research were certified Mekongga variety. Before seedling, seeds were broiled for 1 x 24 hours and then sowed within tray (size of $36 \mathrm{~cm} \mathrm{x} 27 \mathrm{~cm} \mathrm{x}$ $4,5 \mathrm{~cm}$ ) for 14 days (Figure 1). The next step was that the seedlings were transported to the rice field and transplanted.

The rice was planted by using lined up system ('jajar legowo' 2:1) with a planting distance of $50 \mathrm{~cm} \mathrm{x} 25 \mathrm{~cm} \mathrm{x}$ $12.5 \mathrm{~cm}$. All of the rice plot treatments were fertilized with liquid compost extract once in two weeks at a dose of 2 L.ha ${ }^{-1}$ and the manure fertilizer at a dose of 1 ton.ha ${ }^{-1}$. Compost extract was produced according to the method of Suwandi et al. (2012).

Treatment plots were designed into four treatments (four plots) which consisted of rice plots surrounded by (i) refugia, (ii) vegetables, (iii) untreated (without refugia, vegetable or insecticide), and (iv) control sprayed with synthetic insecticide, respectively. Every plot had an area of $800 \mathrm{~m}^{2}$ and was divided into five sub-plots as replications.

Refugia and vegetable planting was conducted 30 days prior to rice planting so that refugia got bloomed when rice entered a vegetative stage at 14 days after transplanting (dat). The rice plots surrounded by refugia used four species of refugia flower consisting of zinnia or paper flower (Zinnia sp.), tagetes (Tagetes erecta), thinkin (Cosmo caudatus), and sesame (Sesamum) (Figure 2). The rice plots surrounded by vegetables used four seasonal vegetable species consisting of long beans ( $V$. sinensis), cucumber ( $C$. sativus), bitter melon (M. charantia), and squash ( $L$. acutangula) planted at the rice field embankment. The vegetable planting distance (distance between planting holes) was as follows: $30 \mathrm{~cm}, 60 \mathrm{~cm}, 30$ $\mathrm{cm}$, and $40 \mathrm{~cm}$ for long beans, bitter melon, squash, and cucumber respectively. The refugia planting distance (distance between planting holes) was $15 \mathrm{~cm}$, but refugia had two rows planting at rice field embankments. The untreated rice plots (without refugia, vegetables, and insecticide application) was the one having no plants in the vicinity of rice field embankments, whereas the rice plots sprayed with synthetic insecticide were also not surrounded by refugia or vegetables (Figure 2). The synthetic insecticide used in this research had abamectin active ingredients according to the recommended dose.

\section{Arthropods sampling}

Arthropod sampling was conducted every week for one planting season and started when rice reached the age of 14 until 84 days after transplanting (11 observations). The arthropods sampling was carried out in early morning from 06.00 to 07.00 a.m. The arthropods were collected using sweep nets (net handgrip length of $100 \mathrm{~cm}$, length of $75 \mathrm{~cm}$ and diameter of $30 \mathrm{~cm}$ ) based on the methods of Jayakumar and Sankari (2010) and Janzen (2013). The swinging net was purposively touched on rice stem according to the method of Masika et al. (2017) in order to drag insects that attached on the rice stems and leaves at rice lower canopy. The arthropods were collected by swinging net once by using double swings that form a straight line at a depth of $30 \mathrm{~cm}$ toward the rice canopy interior.

The caught arthropods were subsequently cleaned and put into plastic vials containing $70 \%$ alcohol, labeled, and taken to the Entomology Laboratory, Department of Plant Pests and Diseases, Faculty of Agriculture, Universitas Sriwijaya, South Sumatra, Indonesia for identification by Dr. Chandra Irsan, an insect taxonomist. The spider visual identification was conducted by using the reference of Barrion and Litsinger (1995), and insect visual identification was conducted by using the references of Heinrichs (1994), Kalshoven (1981), and McAlpine et al. (1987). All the identified arthropods were grouped into guilds, and their number of individuals of each species was counted.

\section{Data analysis}

Data of abundance (or the number of individuals) of herbivores, spiders, and predatory insects among the treatments were analyzed by using analysis of variance (ANOVA). If there were differences among the data of each treatment, they were separated using Tukey's Honestly Significant Different (HSD) test at 5\%. The data analysis was calculated using the program software of SAS University Edition 2.7 9.4 M5. The data of arthropod abundance were also used to analyze the species diversity by using the Shannon index (H'). The diversity level was also evaluated by the Evenness index (J') derived from the Shannon function, and Berger-Parker dominance biodiversity indices. Data of arthropod abundance were also subsequently grouped according to guild consisting of herbivore (or phytophagous insects), predator, parasitoid, and neutral insects, and reported graphically.

\section{RESULTS AND DISCUSSION}

\section{Arthropods species}

Twenty-five herbivore species of 12 families were found during one planting season of rice. The most dominant herbivore found in this research were Nilaparvata lugens, Leptocorisa acuta, Valanga nigricornis, Oxya chinensis, and Acrida turita (Figure 3). 


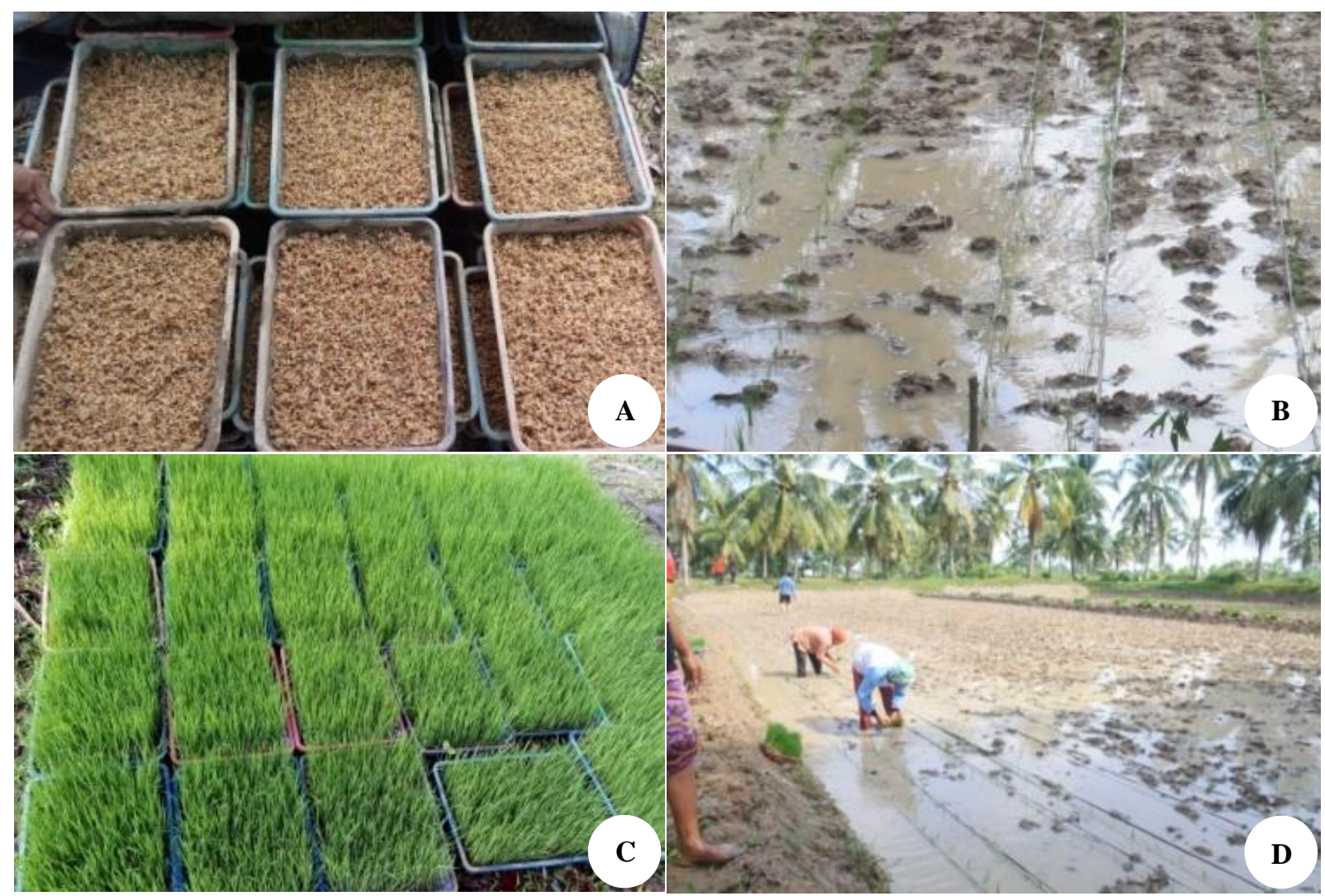

Figure 1. Field preparation: seeds broiled for 1 x 24 hours (A), seeds sowed within trays (B), rice transplanted by using 'jajar legowo' $2: 1(\mathrm{C}, \mathrm{D})$

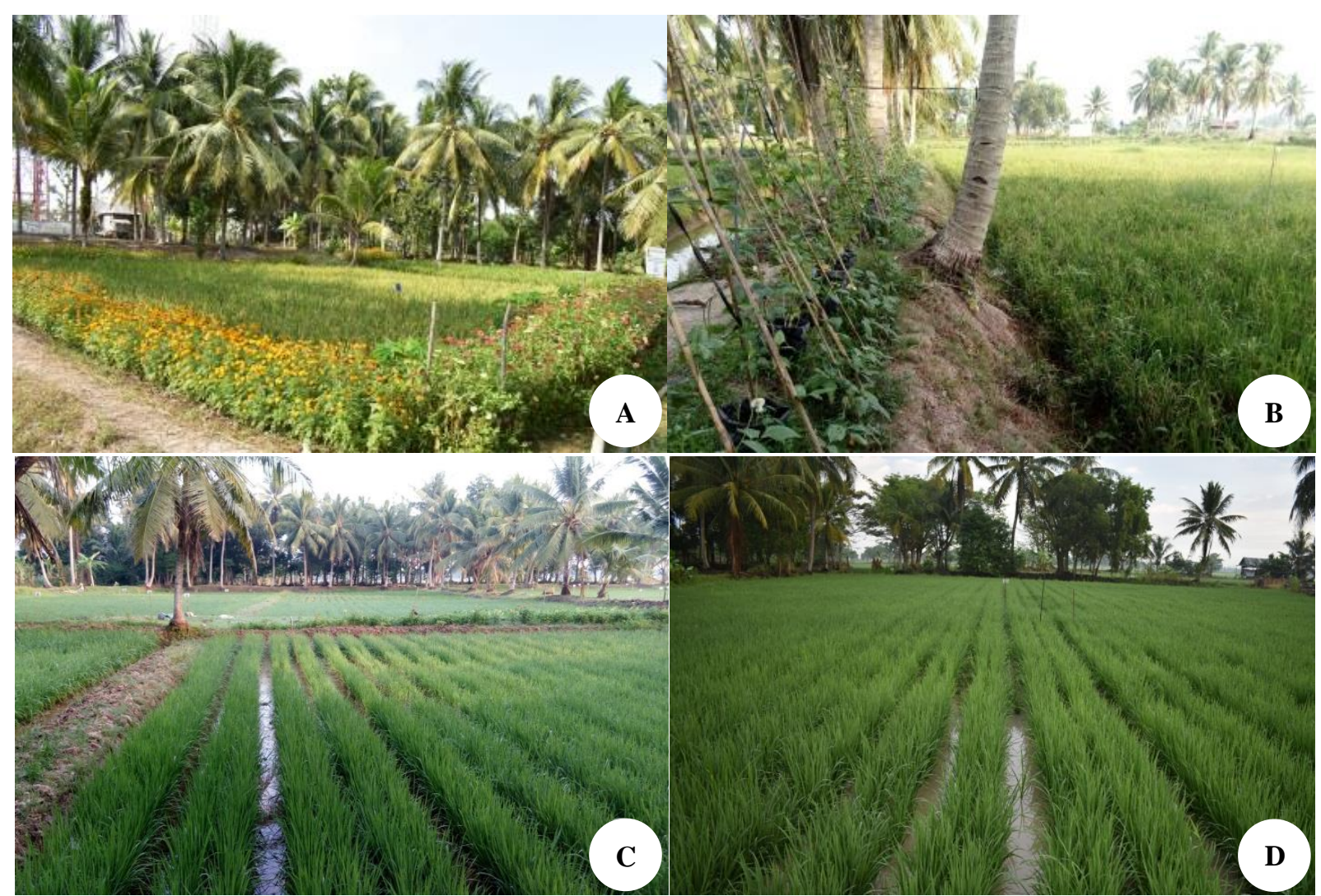

Figure 2. Plots for treatments: rice surrounded by refugia (A), vegetables (B), untreated (C), and insecticide application (D) 


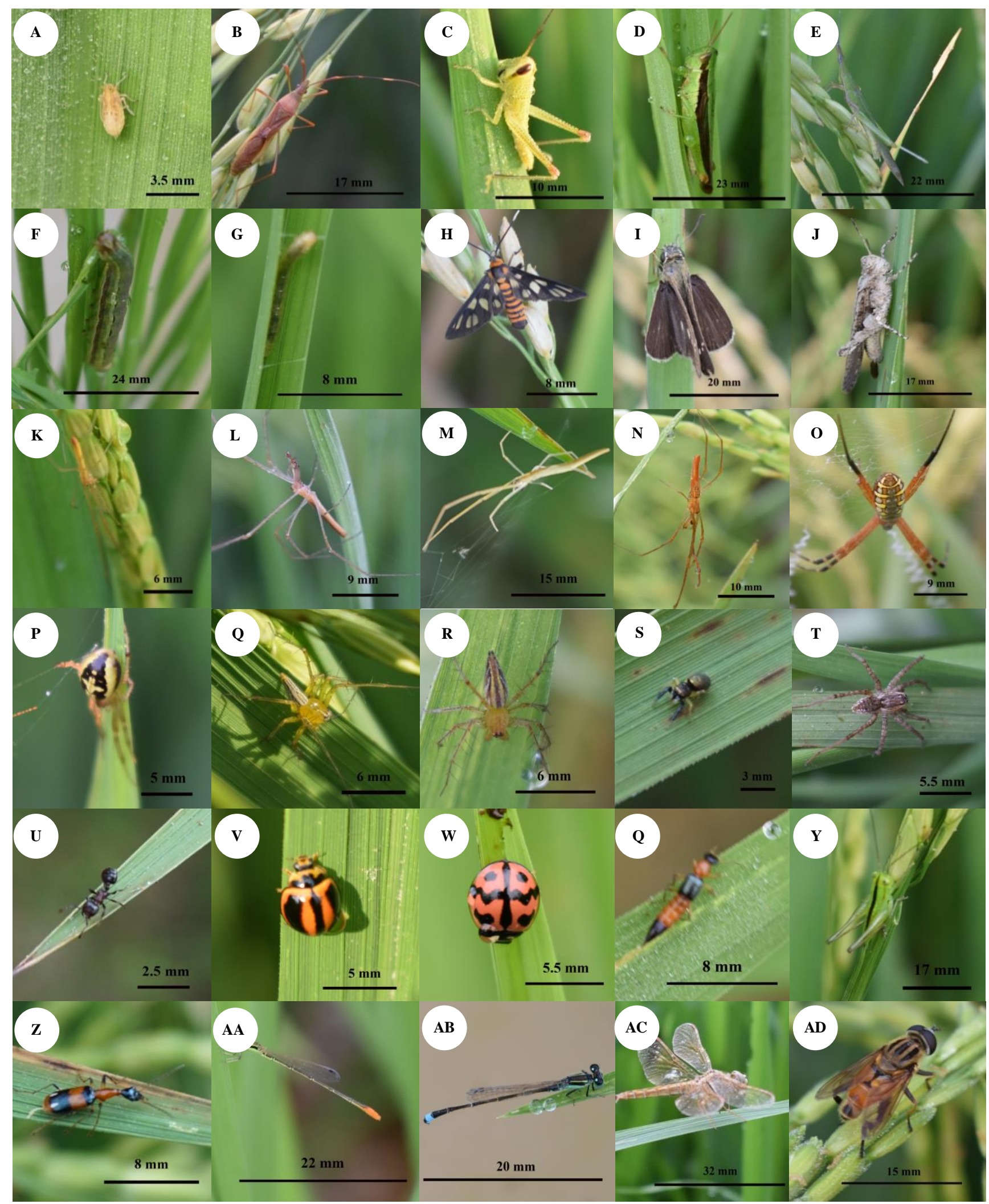

Figure 3. Dominant arthropod species found on the rice field: Nilaparvata lugens (A), Leptocorisa acuta (B), Valanga nigricornis (C), Oxya chinensis (D), Acrida turita (E), Spodoptera sp. (F), Cnaphalocrosis medinalis (G), Amata nigriceps (H), Pelopidas sp. (I), Tetrix subulata $(\mathrm{J})$, Tetragnatha virescens $(\mathrm{K})$, T. mandibulata $(\mathrm{L})$, T. javana $(\mathrm{M})$, T. maxilosa $(\mathrm{N})$, Argiope catenulata $(\mathrm{O})$, Araneidae $(\mathrm{P})$, Oxyopes matiensis (Q), O. javanus (R), Salticidae (S), Pardosa pseudoannulata (T), Odontoponera transversa (U), Micrapis inops (V), Menochilus sexmaculatus (W), Paederus fuscipes (X), Conocephalus longipennis (Y), Ophionea nigrofasciata (Z), Agriocnemis sp. (AA), Argia sp. (AB), Pantala sp. (AC), and Volucella sp. (AD) 

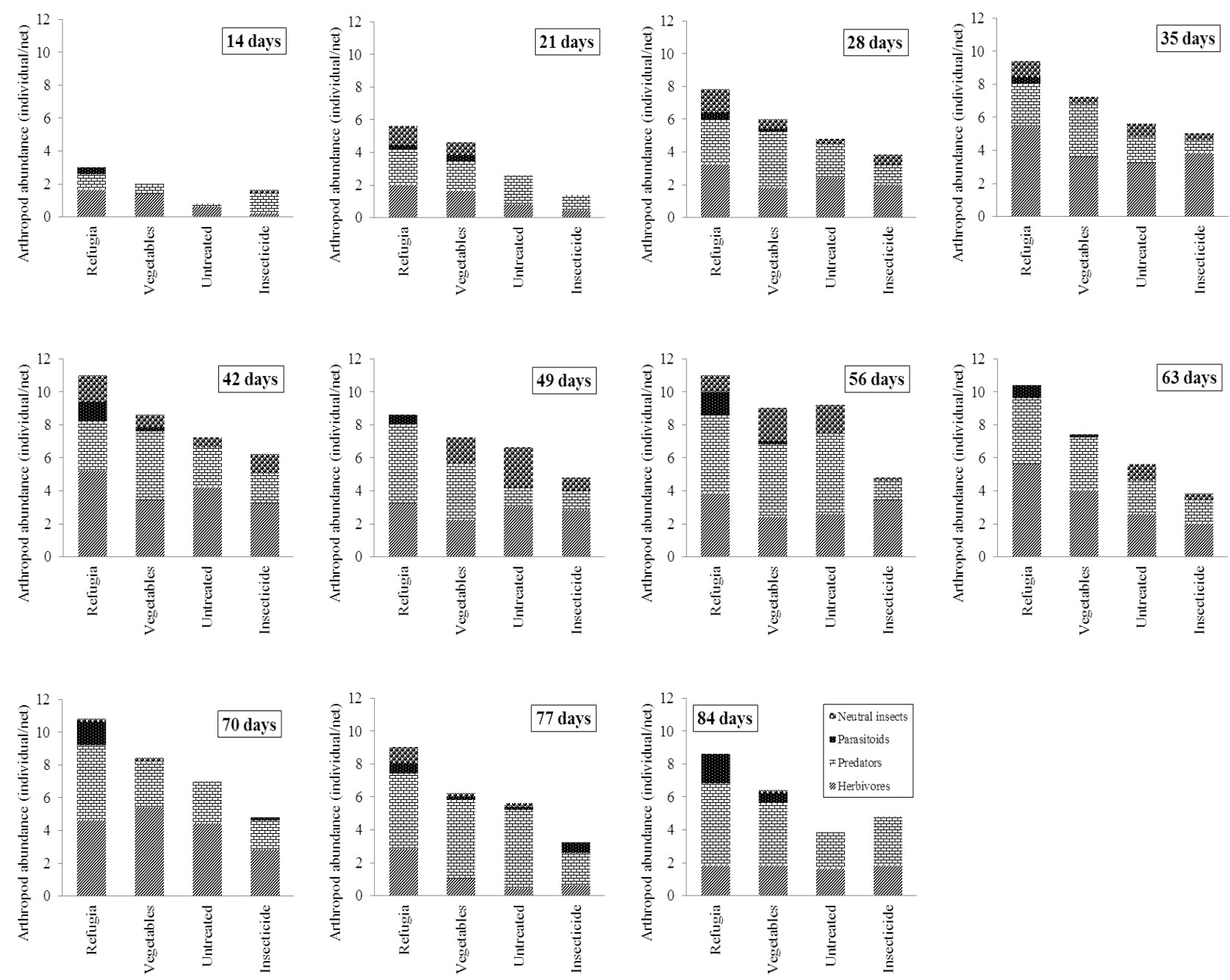

Figure 4. Arthropod abundance on the rice plot surrounded by refugia, vegetables, untreated, and insecticide application

Spiders having 34 species of eight families were dominated by Tetragnatha virescens, $T$. javana, $T$. mandibulata, T. maxilosa, and Argiope catenulata. Predatory insects having 24 species of 12 families were also found in this research and the dominant species of predatory insects were Odontoponera transversa, Micrapis inops, Menochilus sexmaculatus, Paederus fuscipes, and Conocephalus longipennis.

\section{Herbivore abundance}

The highest abundance or population of $N$. lugens, $S$. furcifera, L. acuta, S. litura, and A. turrita during one planting season was found on rice surrounded by refugia and significantly different from the population of those on rice sprayed with synthetic insecticide (Table 1). The highest population of the families of Delphacidae, Alydidae, Noctuidae, and Acrididae was found on rice surrounded by refugia, whereas the lowest population was found on rice sprayed with synthetic insecticide. The application of synthetic insecticide during the vegetative stage of rice significantly decreased the herbivore population.
When the rice reached the age of 14-28 days after transplanting, the herbivore population was still lower on the rice sprayed with synthetic insecticide than that of other treatment plots (Figure 4). After this period, the herbivore population tended to increase on the rice sprayed with synthetic insecticide. Whereas on the other plots (refugia, vegetables, and untreated), the population of herbivore tended to decrease continuously and even when the rice reached the age of 49-56 days after transplanting the rice surrounded by refugia and vegetables, the abundance of predatory arthropods was higher than that of herbivore population.

\section{The abundance of spider and predatory insects}

The highest abundance of $T$. mandibulata, $T$. vermiformis, and Bathyphantes tagalogensis was found on the rice surrounded by refugia and vegetables, and it was significantly different from those on the rice sprayed with synthetic insecticide and untreated rice (Table 2). The total number of spider abundance was also higher on the rice surrounded by refugia and vegetables. The abundance of 
predatory insects of Coccinellidae, especially Micraspis inops, was higher on the rice surrounded by refugia and untreated rice, and it was significantly different from the abundance of rice plots sprayed with synthetics insecticide and the ones surrounded by refugia and vegetables (Table 3). However, the total number of predatory insect abundance was higher on the rice surrounded by refugia, vegetables, and untreated rice, and it was significantly different from those sprayed with synthetic insecticide.

\section{Species diversity of predatory arthropods}

At 14 up to 84 days after transplanting, the spider species diversity on the rice surrounded by refugia tended to be higher than that of other rice plots (Table 4). From the initial to final observation, the total magnitude showed that the highest spider abundance was found on the rice surrounded by refugia, whereas the lowest one was found on the one sprayed with synthetic insecticide. A similar trend was also found on species diversity of predatory insects which tended to be high on the rice surrounded by refugia, whereas a lower species diversity was found on that sprayed with synthetic insecticide (Table 5).

\section{Comparison of arthropod abundance according to guilds}

At 14 days after transplanting, the lowest herbivore abundance was found on the rice sprayed with synthetic insecticide, whereas a higher herbivore abundance was found on other plots (Figure 4). At 21 up to 28 days after transplanting, the abundance of predatory arthropods was higher than that of parasitoids and herbivores. The peak increase of herbivore abundance and the decrease of predatory insect abundance occurred to the rice from 35 to 42 days after transplanting, and subsequently, predatory arthropod abundance started to increase, and it was higher than herbivore abundance. Prior to the harvest at 75 to 84 days after transplanting, the abundance of predatory arthropods sharply increased, and the herbivore abundance drastically decreased. The arthropod abundance (herbivores, predators, parasitoids, and neutral insects) on the rice surrounded by refugia tended to be higher than that of other treatment plots, whereas the lowest abundance tended to be found on the rice plots sprayed with synthetic insecticide.
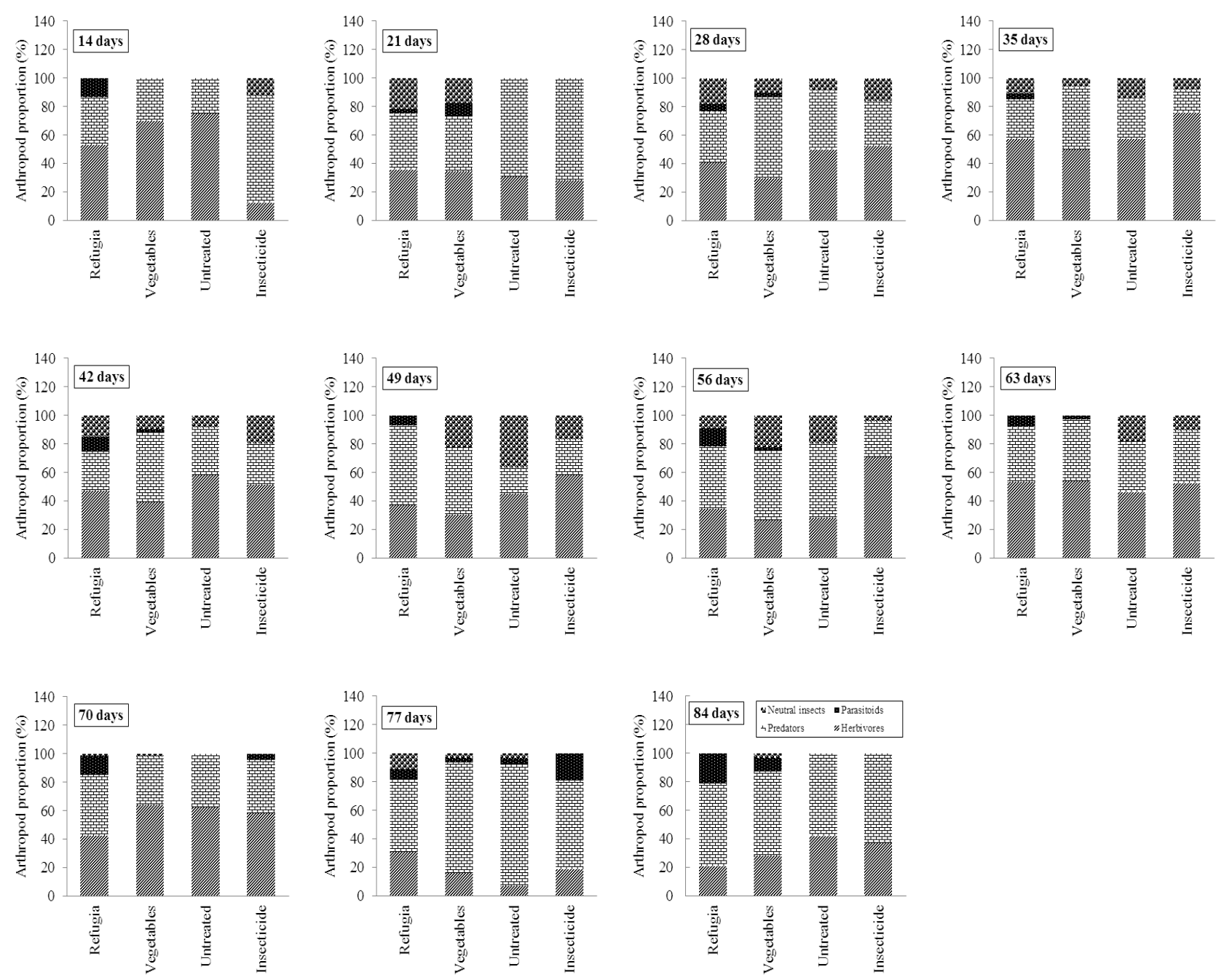

Figure 5. The proportion of arthropods grouped into guilds (herbivore, predator, parasitoid, and neutral insects) on the rice plot surrounded by refugia, vegetables, untreated, and insecticide application 
Table 1. Population or abundance of herbivores inhabiting the rice plot surrounded by refugia, vegetables, untreated, and insecticide application

\begin{tabular}{|c|c|c|c|c|c|c|c|}
\hline \multirow[b]{2}{*}{ Ordo/family/species } & \multicolumn{7}{|c|}{ Mean of population (individual/nets) } \\
\hline & $\begin{array}{c}\text { Rice- } \\
\text { refugia }\end{array}$ & $\begin{array}{c}\text { Rice- } \\
\text { vegetable }\end{array}$ & $\begin{array}{c}\text { Rice- } \\
\text { untreated }\end{array}$ & $\begin{array}{c}\text { Rice- } \\
\text { insecticide }\end{array}$ & $\begin{array}{c}\text { F- } \\
\text { value }\end{array}$ & $\begin{array}{c}\text { P value } \\
(0.05)\end{array}$ & $\begin{array}{c}\text { Tukey's } \\
\text { HSD test }\end{array}$ \\
\hline HEMIPTERA & $1.58^{\mathrm{b}}$ & $1.16^{\mathrm{ab}}$ & $0.75^{\mathrm{a}}$ & $0.78^{\mathrm{a}}$ & 5.84 & 0.01 & 0.27 \\
\hline Delphacidae & $0.95^{\mathrm{b}}$ & $0.69^{a b}$ & $0.51^{\mathrm{ab}}$ & $0.25^{\mathrm{a}}$ & 5.01 & 0.02 & 0.26 \\
\hline Nilavarpata lugens & $0.8^{\mathrm{b}}$ & $0.62^{\mathrm{ab}}$ & $0.47^{\mathrm{ab}}$ & $0.18^{\mathrm{a}}$ & 3.61 & 0.05 & 0.29 \\
\hline Sogatella furcifera & $0.15^{\mathrm{a}}$ & $0.07^{\mathrm{a}}$ & $0.04^{\mathrm{a}}$ & $0.07^{\mathrm{a}}$ & 1.08 & 0.39 & \\
\hline Ciccadelidae & $0^{\mathrm{a}}$ & $0^{\mathrm{a}}$ & $0.02^{\mathrm{a}}$ & $0^{\mathrm{a}}$ & 1 & 0.43 & \\
\hline Cicadulina bipunctata & $0^{\mathrm{a}}$ & $0^{\mathrm{a}}$ & $0.02^{\mathrm{a}}$ & $0^{\mathrm{a}}$ & 1 & 0.43 & \\
\hline Coreidae & $0^{\mathrm{a}}$ & $0.04^{\mathrm{a}}$ & $0^{\mathrm{a}}$ & $0.04^{\mathrm{a}}$ & 0.62 & 0.62 & \\
\hline Cletus trigonus & $0^{\mathrm{a}}$ & $0.04^{\mathrm{a}}$ & $0^{\mathrm{a}}$ & $0.04^{\mathrm{a}}$ & 0.62 & 0.62 & \\
\hline Alydidae & $0.64^{\mathrm{b}}$ & $0.44^{\mathrm{ab}}$ & $0.22^{\mathrm{a}}$ & $0.49^{\mathrm{ab}}$ & 5.50 & 0.01 & 0.17 \\
\hline Leptocorisa acuta & $0.64^{\mathrm{b}}$ & $0.44^{\mathrm{ab}}$ & $0.22^{\mathrm{a}}$ & $0.49^{\mathrm{ab}}$ & 5.50 & 0.01 & 0.17 \\
\hline LEPIDOPTERA & $0.22^{\mathrm{a}}$ & $0.27^{\mathrm{a}}$ & $0.27^{\mathrm{a}}$ & $0.11^{\mathrm{a}}$ & 1.05 & 0.41 & \\
\hline Noctuidae & $0.05^{\mathrm{a}}$ & $0.18^{\mathrm{b}}$ & $0.04^{\mathrm{a}}$ & $0.02^{\mathrm{a}}$ & 6.66 & 0.01 & 0.08 \\
\hline Spodoptera sp. & $0.05^{\mathrm{ab}}$ & $0.15^{\mathrm{b}}$ & $0.04^{\mathrm{a}}$ & $0^{\mathrm{a}}$ & 5.97 & 0.01 & 0.06 \\
\hline Naranga sp. & $0^{\mathrm{a}}$ & $0.04^{\mathrm{a}}$ & $0^{\mathrm{a}}$ & $0.02^{\mathrm{a}}$ & 0.68 & 0.58 & \\
\hline Pyralidae & $0.07^{\mathrm{a}}$ & $0.05^{\mathrm{a}}$ & $0.09^{\mathrm{a}}$ & $0.09^{\mathrm{a}}$ & 0.26 & 0.85 & \\
\hline Cnaphalocrosis medinalis & $0.05^{\mathrm{a}}$ & $0.05^{\mathrm{a}}$ & $0.04^{\mathrm{a}}$ & $0.04^{\mathrm{a}}$ & 0.11 & 0.95 & \\
\hline Scirpophaga incertulas & $0.02^{\mathrm{a}}$ & $0^{\mathrm{a}}$ & $0.05^{\mathrm{a}}$ & $0.05^{\mathrm{a}}$ & 1.62 & 0.24 & \\
\hline Erebidae & $0.04^{\mathrm{a}}$ & $0.04^{\mathrm{a}}$ & $0.13^{\mathrm{a}}$ & $0^{\mathrm{a}}$ & 1.21 & 0.35 & \\
\hline Amata nigriceps & $0.04^{\mathrm{a}}$ & $0.04^{\mathrm{a}}$ & $0.13^{\mathrm{a}}$ & $0^{\mathrm{a}}$ & 1.21 & 0.35 & \\
\hline Hesperiidae & $0.02^{\mathrm{a}}$ & $0^{\mathrm{a}}$ & $0^{\mathrm{a}}$ & $0^{\mathrm{a}}$ & 1 & 0.43 & \\
\hline Pelopidas sp. & $0.02^{\mathrm{a}}$ & $0^{\mathrm{a}}$ & $0^{\mathrm{a}}$ & $0^{\mathrm{a}}$ & 1 & 0.43 & \\
\hline Nymphalidae & $0.04^{\mathrm{a}}$ & $0^{\mathrm{a}}$ & $0.02^{\mathrm{a}}$ & $0^{\mathrm{a}}$ & 0.68 & 0.58 & \\
\hline Melanitis leda & $0.04^{\mathrm{a}}$ & $0^{\mathrm{a}}$ & $0.02^{\mathrm{a}}$ & $0^{\mathrm{a}}$ & 0.68 & 0.58 & \\
\hline COLEOPTERA & $0.09^{\mathrm{a}}$ & $0.11^{\mathrm{a}}$ & $0.07^{\mathrm{a}}$ & $0.05^{\mathrm{a}}$ & 0.17 & 0.92 & \\
\hline Chrysomelidae & $0.09^{\mathrm{a}}$ & $0.11^{\mathrm{a}}$ & $0.07^{\mathrm{a}}$ & $0.05^{\mathrm{a}}$ & 0.17 & 0.92 & \\
\hline Chrysolina coerulans & $0.07^{\mathrm{a}}$ & $0.04^{\mathrm{a}}$ & $0.07^{\mathrm{a}}$ & $0.00^{\mathrm{a}}$ & 0.63 & 0.61 & \\
\hline Dactylispa bayoni & $0^{\mathrm{a}}$ & $0^{\mathrm{a}}$ & $0^{\mathrm{a}}$ & $0.02^{\mathrm{a}}$ & 1 & 0.43 & \\
\hline Chaetocnema sp. & $0.02^{\mathrm{a}}$ & $0^{\mathrm{a}}$ & $0^{\mathrm{a}}$ & $0^{\mathrm{a}}$ & 1 & 0.43 & \\
\hline Dicladispa armigera & $0^{\mathrm{a}}$ & $0.02^{\mathrm{a}}$ & $0^{\mathrm{a}}$ & $0^{\mathrm{a}}$ & 1 & 0.43 & \\
\hline Aulacophora frontalis & $0^{\mathrm{a}}$ & $0.04^{\mathrm{a}}$ & $0^{\mathrm{a}}$ & $0.04^{\mathrm{a}}$ & 0.88 & 0.48 & \\
\hline Charidotella sp. & $0^{\mathrm{a}}$ & $0.02^{\mathrm{a}}$ & $0^{\mathrm{a}}$ & $0^{\mathrm{a}}$ & 1 & 0.43 & \\
\hline ORTHOPTERA & $1.51^{\mathrm{b}}$ & $0.89^{\mathrm{a}}$ & $1.24^{\mathrm{ab}}$ & $1.04^{\mathrm{ab}}$ & 3.63 & 0.05 & 0.22 \\
\hline Tettigonidae & $0^{\mathrm{a}}$ & $0^{\mathrm{a}}$ & $0.04^{\mathrm{a}}$ & $0^{\mathrm{a}}$ & 1 & 0.43 & \\
\hline Tetrix subulata & $0^{\mathrm{a}}$ & $0^{\mathrm{a}}$ & $0.04^{\mathrm{a}}$ & $0^{\mathrm{a}}$ & 1 & 0.43 & \\
\hline Acrididae & $1.49^{\mathrm{b}}$ & $0.89^{\mathrm{a}}$ & $1.16^{\mathrm{ab}}$ & $1.04^{\mathrm{ab}}$ & 3.64 & 0.04 & 0.21 \\
\hline Valanga nigricornis & $1.18^{\mathrm{a}}$ & $0.69^{\mathrm{a}}$ & $1.15^{\mathrm{a}}$ & $0.87^{\mathrm{a}}$ & 2.29 & 0.13 & \\
\hline Oxya chinensis & $0.2^{\mathrm{a}}$ & $0.07^{\mathrm{a}}$ & $0.02^{\mathrm{a}}$ & $0.11^{\mathrm{a}}$ & 1.67 & 0.23 & \\
\hline Acrida turrita & $0.11^{\mathrm{b}}$ & $0.13^{\mathrm{b}}$ & $0.00^{\mathrm{a}}$ & $0.05^{\mathrm{ab}}$ & 6.11 & 0.01 & 0.06 \\
\hline Gryllidae & $0.02^{\mathrm{a}}$ & $0^{\mathrm{a}}$ & $0.04^{\mathrm{a}}$ & $0^{\mathrm{a}}$ & 0.68 & 0.58 & \\
\hline Gryllus sp. & $0.02^{\mathrm{a}}$ & $0^{\mathrm{a}}$ & $0.04^{\mathrm{a}}$ & $0^{\mathrm{a}}$ & 0.681 & 0.58 & \\
\hline Diptera & $0.11^{\mathrm{a}}$ & $0.16^{\mathrm{a}}$ & $0.02^{\mathrm{a}}$ & $0.11^{\mathrm{a}}$ & 1.93 & 0.18 & \\
\hline Cecidomyiidae & $0.11^{\mathrm{a}}$ & $0.16^{\mathrm{a}}$ & $0.02^{\mathrm{a}}$ & $0.11^{\mathrm{a}}$ & 1.93 & 0.18 & \\
\hline Orselia sp. & $0.11^{\mathrm{a}}$ & $0.16^{\mathrm{a}}$ & $0.02^{\mathrm{a}}$ & $0.11^{\mathrm{a}}$ & 1.93 & 0.18 & \\
\hline THYSANOPTERA & $0.05^{\mathrm{a}}$ & $0^{\mathrm{a}}$ & $0^{\mathrm{a}}$ & $0^{\mathrm{a}}$ & 2.29 & 0.13 & \\
\hline Thripidae & $0.05^{\mathrm{a}}$ & $0^{\mathrm{a}}$ & $0^{\mathrm{a}}$ & $0^{\mathrm{a}}$ & 2.29 & 0.13 & \\
\hline Thrips sp. & $0.05^{\mathrm{a}}$ & $0^{\mathrm{a}}$ & $0^{\mathrm{a}}$ & $0^{\mathrm{a}}$ & 2.29 & 0.13 & \\
\hline Total abundance $(\mathrm{N})$ & $3.56^{\mathrm{b}}$ & $2.6^{\mathrm{ab}}$ & $2.35^{\mathrm{a}}$ & $2.09^{\mathrm{a}}$ & 5.14 & 0.02 & 0.34 \\
\hline
\end{tabular}

Note: Values within a row followed by the same letters were not significantly different at $\mathrm{P}<0.05$ according to Tukey's HSD test

The initial observation period at 14 to 21 days after transplanting showed that the herbivore proportion on the rice sprayed with synthetic insecticide was lower than the proportion of predators, parasitoids, and neutral insects, whereas the highest herbivore proportion was found in the rice surrounded by refugia (Figure 5). Subsequently, herbivore proportion on the rice sprayed with synthetic insecticide continually increased and got higher than the predator proportion. However, the predator and parasitoid proportion on the rice surrounded by refugia and vegetable continually increased and got higher than the herbivore proportion up to before the harvest.

\section{Discussion}

This research results showed that refugia and vegetables planted on rice field embankments surrounded by rice were 
capable of increasing the abundance and species diversity of predatory arthropods (spiders and predatory insects) on the rice plot, whereas synthetic insecticide application did not only suppress herbivore population but also decreased the abundance of natural enemies of pest insects and other useful arthropods, such as spiders, predatory insects, parasitoids, neutral insects or pollinating insects.

Refugia as habitat were capable of providing microclimate in the form of high relative humidity and shady condition that appropriate for natural enemies habitat either predatory arthropods or parasitoids (McCabe et al. 2017). Refugia as habitat were not only required during rice growth in a rice field but also had functioned as a sink for arthropods during a rice harvest and would become arthropod source during the initial rice planting in rice fields (Baba et al. 2018). It was evident that Zinnia sp. as habitat was inhabited by spiders of the families of Araneidae, Oxyopidae, and Pisauridae (Desai et al. 2017). The spider (Oxyopidae), predatory insects (Coccinellidae, Syrphidae, and Geocoridae), parasitoid and pollinating insects of Hymenoptera and Lepidoptera were found in large numbers inhabiting $T$. erecta as niche (Ganai et al. 2017).

Table 2. Abundance of spiders inhabiting the rice plot surrounded by refugia, vegetables, untreated, and insecticide application

\begin{tabular}{|c|c|c|c|c|c|c|c|}
\hline \multirow[b]{2}{*}{ Family and species } & \multicolumn{7}{|c|}{ Mean of abundance (individual/nets) } \\
\hline & $\begin{array}{c}\text { Rice- } \\
\text { refugia }\end{array}$ & $\begin{array}{c}\text { Rice- } \\
\text { vegetable }\end{array}$ & $\begin{array}{c}\text { Rice- } \\
\text { untreated }\end{array}$ & $\begin{array}{c}\text { Rice- } \\
\text { insecticide }\end{array}$ & F-value & $\begin{array}{c}\text { P value } \\
(0.05)\end{array}$ & $\begin{array}{c}\text { Tukey's } \\
\text { HSD test }\end{array}$ \\
\hline Lycosidae & $0.07^{\mathrm{a}}$ & $0.02^{\mathrm{a}}$ & $0.02^{\mathrm{a}}$ & $0.04^{\mathrm{a}}$ & 0.89 & 0.48 & \\
\hline Pardosa pseudoannulata & $0.07^{\mathrm{a}}$ & $0.02^{\mathrm{a}}$ & $0.02^{\mathrm{a}}$ & $0.04^{\mathrm{a}}$ & 0.89 & 0.48 & \\
\hline Araneidae & $0.20^{\mathrm{b}}$ & $0.15^{\mathrm{ab}}$ & $0.04^{\mathrm{a}}$ & $0.02^{\mathrm{a}}$ & 7.29 & 0.01 & 0.08 \\
\hline Araneus inustus & $0.02^{\mathrm{a}}$ & $0.02^{\mathrm{a}}$ & $0^{\mathrm{a}}$ & $0^{\mathrm{a}}$ & 1 & 0.43 & \\
\hline Cylosa insulana & $0.05^{\mathrm{a}}$ & $0.07^{\mathrm{a}}$ & $0.02^{\mathrm{a}}$ & $0.02^{\mathrm{a}}$ & 1.12 & 0.38 & \\
\hline Cyrtophora koronadalensis & $0.05^{\mathrm{a}}$ & $0^{\mathrm{a}}$ & $0^{\mathrm{a}}$ & $0^{\mathrm{a}}$ & 2.29 & 0.13 & \\
\hline Neoscona theisi & $0.02^{\mathrm{a}}$ & $0^{\mathrm{a}}$ & $0^{\mathrm{a}}$ & $0^{\mathrm{a}}$ & 1 & 0.43 & \\
\hline Argiope catenulata & $0.05^{\mathrm{a}}$ & $0.05^{\mathrm{a}}$ & $0.02^{\mathrm{a}}$ & $0^{\mathrm{a}}$ & 1.14 & 0.38 & \\
\hline Tetragnathidae & $1.55^{\mathrm{c}}$ & $1.55^{\mathrm{c}}$ & $0.91^{\mathrm{ab}}$ & $0.76^{\mathrm{a}}$ & 7.72 & 0 & 0.24 \\
\hline Tetragnatha javana & $0.16^{\mathrm{a}}$ & $0.2^{\mathrm{a}}$ & $0.15^{\mathrm{a}}$ & $0.22^{\mathrm{a}}$ & 0.38 & 0.77 & \\
\hline Tetragnatha virescens & $0.73^{\mathrm{a}}$ & $0.82^{\mathrm{a}}$ & $0.51^{\mathrm{a}}$ & $0.4^{\mathrm{a}}$ & 2.38 & 0.12 & \\
\hline Tetragnatha mandibulata & $0.35^{\mathrm{b}}$ & $0.22^{\mathrm{ab}}$ & $0.16^{\mathrm{ab}}$ & $0.11^{\mathrm{a}}$ & 4.26 & 0.03 & 0.12 \\
\hline Tetragnatha ilavaca & $0^{\mathrm{a}}$ & $0^{\mathrm{a}}$ & $0^{\mathrm{a}}$ & $0.02^{\mathrm{a}}$ & 1 & 0.43 & \\
\hline Tetragnatha maxillosa & $0.16^{\mathrm{a}}$ & $0.07^{\mathrm{a}}$ & $0.05^{\mathrm{a}}$ & $0^{\mathrm{a}}$ & 2.18 & 0.14 & \\
\hline Tetragnatha vermiformis & $0.09^{\mathrm{ab}}$ & $0.22^{\mathrm{b}}$ & $0.04^{\mathrm{ab}}$ & $0.02^{\mathrm{a}}$ & 4.09 & 0.03 & 0.12 \\
\hline Tetragnatha Okumae & $0.02^{\mathrm{a}}$ & $0.02^{\mathrm{a}}$ & $0^{\mathrm{a}}$ & $0^{\mathrm{a}}$ & 0.62 & 0.62 & \\
\hline Tetragnatha iwahigensis & $0.02 \mathrm{a}$ & $0 \mathrm{a}$ & $0 \mathrm{a}$ & $0 \mathrm{a}$ & 1 & 0.43 & \\
\hline Tetragnatha natans & $0.02^{\mathrm{a}}$ & $0^{\mathrm{a}}$ & $0^{\mathrm{a}}$ & $0^{\mathrm{a}}$ & 1 & 0.43 & \\
\hline Linyphiidae & $0.16^{\mathrm{a}}$ & $0.15^{\mathrm{a}}$ & $0.05^{\mathrm{a}}$ & $0.15^{\mathrm{a}}$ & 1 & 0.43 & \\
\hline Bathyphantes tagalogensis & $0.13^{\mathrm{a}}$ & $0.09^{\mathrm{a}}$ & $0^{\mathrm{a}}$ & $0.04^{\mathrm{a}}$ & 2.97 & 0.07 & \\
\hline Bathyphantes sp. & $0^{\mathrm{a}}$ & $0.02^{\mathrm{a}}$ & $0.04^{\mathrm{a}}$ & $0.05^{\mathrm{a}}$ & 0.88 & 0.48 & \\
\hline Erigone bifurca & $0^{\mathrm{a}}$ & $0^{\mathrm{a}}$ & $0^{\mathrm{a}}$ & $0.02^{\mathrm{a}}$ & 1 & 0.43 & \\
\hline Enoplognatha sp. & $0.04^{\mathrm{a}}$ & $0.04^{\mathrm{a}}$ & $0^{\mathrm{a}}$ & $0.04^{\mathrm{a}}$ & 0.83 & 0.5 & \\
\hline Unknown & $0^{\mathrm{a}}$ & $0^{\mathrm{a}}$ & $0.02^{\mathrm{a}}$ & $0^{\mathrm{a}}$ & 1 & 0.43 & \\
\hline Oxyopidae & $0.15^{\mathrm{a}}$ & $0.11^{\mathrm{a}}$ & $0.09^{\mathrm{a}}$ & $0.04^{\mathrm{a}}$ & 0.8 & 0.52 & \\
\hline Oxyopes javanus & $0.04^{\mathrm{a}}$ & $0.02^{\mathrm{a}}$ & $0^{\mathrm{a}}$ & $0.04^{\mathrm{a}}$ & 0.69 & 0.58 & \\
\hline Oxyopes matiensis & $0.09^{\mathrm{a}}$ & $0.09^{a}$ & $0.09^{\mathrm{a}}$ & $0^{\mathrm{a}}$ & 0.73 & 0.56 & \\
\hline Oxyopes pingasus & $0.02^{\mathrm{a}}$ & $0^{\mathrm{a}}$ & $0^{\mathrm{a}}$ & $0^{\mathrm{a}}$ & 1 & 0.43 & \\
\hline Theridiidae & $0.02^{\mathrm{a}}$ & $0.02^{\mathrm{a}}$ & $0^{\mathrm{a}}$ & $0^{\mathrm{a}}$ & 1 & 0.43 & \\
\hline Theridion sp. & $0.02^{\mathrm{a}}$ & $0.02^{\mathrm{a}}$ & $0^{\mathrm{a}}$ & $0^{\mathrm{a}}$ & 1 & 0.43 & \\
\hline Salticidae & $0.11^{\mathrm{a}}$ & $0.09^{\mathrm{a}}$ & $0.07^{\mathrm{a}}$ & $0.09^{\mathrm{a}}$ & 0.13 & 0.94 & \\
\hline Myrmarachne bidentata & $0^{\mathrm{a}}$ & $0.02^{\mathrm{a}}$ & $0^{\mathrm{a}}$ & $0^{\mathrm{a}}$ & 1 & 0.43 & \\
\hline Hyllus maskaranus & $0.02^{\mathrm{a}}$ & $0.02^{\mathrm{a}}$ & $0.05^{\mathrm{a}}$ & $0^{\mathrm{a}}$ & 2.11 & 0.15 & \\
\hline Cosmophasis parangpilota & $0.02^{\mathrm{a}}$ & $0^{\mathrm{a}}$ & $0^{\mathrm{a}}$ & $0^{\mathrm{a}}$ & 1 & 0.43 & \\
\hline Cosmophasis sp. & $0.04^{\mathrm{a}}$ & $0.04^{\mathrm{a}}$ & $0^{\mathrm{a}}$ & $0.02^{\mathrm{a}}$ & 0.82 & 0.51 & \\
\hline Flexipus paykulli & $0^{\mathrm{a}}$ & $0^{\mathrm{a}}$ & $0^{\mathrm{a}}$ & $0.04^{\mathrm{a}}$ & 1 & 0.43 & \\
\hline Harmochirus brachiatus & $0.02^{\mathrm{a}}$ & $0^{\mathrm{a}}$ & $0.02^{\mathrm{a}}$ & $0^{\mathrm{a}}$ & 0.62 & 0.62 & \\
\hline Bianor hoting chiehi & $0^{\mathrm{a}}$ & $0.02^{\mathrm{a}}$ & $0^{\mathrm{a}}$ & $0.02^{\mathrm{a}}$ & 0.62 & 0.62 & \\
\hline Emathis sp. & $0.02^{\mathrm{a}}$ & $0^{\mathrm{a}}$ & $0^{\mathrm{a}}$ & $0^{\mathrm{a}}$ & 1 & 0.43 & \\
\hline Unknown & $0^{\mathrm{a}}$ & $0^{\mathrm{a}}$ & $0^{\mathrm{a}}$ & $0.02^{\mathrm{a}}$ & 1 & 0.43 & \\
\hline Hahniidae & $0^{\mathrm{a}}$ & $0.02^{\mathrm{a}}$ & $0.04^{\mathrm{a}}$ & $0^{\mathrm{a}}$ & 0.68 & 0.58 & \\
\hline Hahnia tuybaana & $0^{\mathrm{a}}$ & $0.02^{\mathrm{a}}$ & $0.04^{\mathrm{a}}$ & $0^{\mathrm{a}}$ & 0.68 & 0.58 & \\
\hline Total abundance $(\mathrm{N})$ & $2.25^{\mathrm{b}}$ & $2.09^{\mathrm{b}}$ & $1.22^{\mathrm{a}}$ & $1.09^{\mathrm{a}}$ & 27.51 & 0 & 0.17 \\
\hline
\end{tabular}

Note: Values within a row followed by the same letters were not significantly different at $\mathrm{P}<0.05$ according to Tukey's HSD test 
Table 3. The abundance of predatory insects inhabiting the rice plot surrounded by refugia, vegetables, untreated, and insecticide application for one planting season

\begin{tabular}{|c|c|c|c|c|c|c|c|}
\hline \multirow[b]{2}{*}{ Family and species } & \multicolumn{7}{|c|}{ Mean of abundance (individual/nets) } \\
\hline & $\begin{array}{c}\text { Rice- } \\
\text { refugia }\end{array}$ & $\begin{array}{c}\text { Rice- } \\
\text { vegetable }\end{array}$ & $\begin{array}{c}\text { Rice- } \\
\text { untreated }\end{array}$ & $\begin{array}{c}\text { Rice- } \\
\text { insecticide }\end{array}$ & F-value & $\begin{array}{c}\text { P value } \\
(0.05)\end{array}$ & $\begin{array}{c}\text { Tukey's } \\
\text { HSD test }\end{array}$ \\
\hline HEMIPTERA & $0.07^{\mathrm{a}}$ & $0.02^{\mathrm{a}}$ & $0.02^{\mathrm{a}}$ & $0.02^{\mathrm{a}}$ & 1.17 & 0.36 & \\
\hline Miridae & $0.07^{\mathrm{a}}$ & $0.02^{\mathrm{a}}$ & $0.02^{\mathrm{a}}$ & $0.02^{\mathrm{a}}$ & 1.17 & 0.36 & \\
\hline Cyrtorhinus lividipennis & $0.04^{\mathrm{a}}$ & $0.02^{\mathrm{a}}$ & $0^{\mathrm{a}}$ & $0.02^{\mathrm{a}}$ & 1.46 & 0.28 & \\
\hline Orthotylus sp. & $0.04^{\mathrm{a}}$ & $0^{\mathrm{a}}$ & $0.02^{\mathrm{a}}$ & $0^{\mathrm{a}}$ & 1.29 & 0.32 & \\
\hline COLEOPTERA & $1.09^{\mathrm{b}}$ & $0.35^{\mathrm{a}}$ & $0.67^{\mathrm{ab}}$ & $0.29^{\mathrm{a}}$ & 12.75 & 0 & 0.20 \\
\hline Carabidae & $0.02^{\mathrm{a}}$ & $0^{\mathrm{a}}$ & $0^{\mathrm{a}}$ & $0^{\mathrm{a}}$ & 1 & 0.43 & \\
\hline Ophionea nigrofasciata & $0.02^{\mathrm{a}}$ & $0^{\mathrm{a}}$ & $0^{\mathrm{a}}$ & $0^{\mathrm{a}}$ & 1 & 0.43 & \\
\hline Staphylinidae & $0.27^{\mathrm{a}}$ & $0.16^{\mathrm{a}}$ & $0.09^{\mathrm{a}}$ & $0.11^{\mathrm{a}}$ & 2.25 & 0.14 & \\
\hline Paederus fuscipes & $0.27^{\mathrm{a}}$ & $0.16^{\mathrm{a}}$ & $0.09^{\mathrm{a}}$ & $0.11^{\mathrm{a}}$ & 2.25 & 0.14 & \\
\hline Coccinelidae & $0.78^{\mathrm{b}}$ & $0.16^{\mathrm{a}}$ & $0.56^{\mathrm{b}}$ & $0.18^{\mathrm{a}}$ & 11.72 & 0 & 0.19 \\
\hline Micraspis inops & $0.38^{\mathrm{b}}$ & $0.07^{\mathrm{a}}$ & $0.25^{\mathrm{ab}}$ & $0.15^{\mathrm{ab}}$ & 4.8 & 0.02 & 0.16 \\
\hline Verania lineata & $0.04^{\mathrm{a}}$ & $0^{\mathrm{a}}$ & $0^{\mathrm{a}}$ & $0^{\mathrm{a}}$ & 1 & 0.43 & \\
\hline Micraspis vincta & $0.02^{\mathrm{a}}$ & $0^{\mathrm{a}}$ & $0.04^{\mathrm{a}}$ & $0^{\mathrm{a}}$ & 1 & 0.43 & \\
\hline Harmonia octomaculata & $0^{\mathrm{a}}$ & $0^{\mathrm{a}}$ & $0.04^{\mathrm{a}}$ & $0^{\mathrm{a}}$ & 1 & 0.43 & \\
\hline Coccinella repanda & $0.05^{\mathrm{a}}$ & $0.02^{\mathrm{a}}$ & $0.02^{\mathrm{a}}$ & $0^{\mathrm{a}}$ & 0.99 & 0.43 & \\
\hline Brumoides suturalis & $0.04^{\mathrm{a}}$ & $0^{\mathrm{a}}$ & $0.04^{\mathrm{a}}$ & $0^{\mathrm{a}}$ & 0.88 & 0.48 & \\
\hline Menochilus sexmaculatus & $0^{\mathrm{a}}$ & $0^{\mathrm{a}}$ & $0.02^{\mathrm{a}}$ & $0^{\mathrm{a}}$ & 1 & 0.43 & \\
\hline Unknown & $0.25^{\mathrm{b}}$ & $0.07^{\mathrm{ab}}$ & $0.16^{\mathrm{ab}}$ & $0.04^{\mathrm{a}}$ & 3.77 & 0.04 & 0.13 \\
\hline Elateridae & $0.02^{\mathrm{a}}$ & $0^{\mathrm{a}}$ & $0^{\mathrm{a}}$ & $0^{\mathrm{a}}$ & 1 & 0.43 & \\
\hline Unknown & $0.02^{\mathrm{a}}$ & $0^{\mathrm{a}}$ & $0^{\mathrm{a}}$ & $0^{\mathrm{a}}$ & 1 & 0.43 & \\
\hline Anthicidae & $0^{\mathrm{a}}$ & $0.02^{\mathrm{a}}$ & $0.02^{\mathrm{a}}$ & $0^{\mathrm{a}}$ & 0.62 & 0.62 & \\
\hline Formicomus sp. & $0^{\mathrm{a}}$ & $0.02^{\mathrm{a}}$ & $0.02^{\mathrm{a}}$ & $0^{\mathrm{a}}$ & 0.62 & 0.62 & \\
\hline ODONATA & $0.04^{\mathrm{a}}$ & $0.09^{\mathrm{a}}$ & $0.04^{\mathrm{a}}$ & $0.05^{\mathrm{a}}$ & 0.66 & 0.59 & \\
\hline Coenagrionidae & $0.04^{\mathrm{a}}$ & $0.07^{\mathrm{a}}$ & $0.04^{\mathrm{a}}$ & $0.04^{\mathrm{a}}$ & 0.52 & 0.68 & \\
\hline Agriocnemis sp. & $0.02^{\mathrm{a}}$ & $0.05^{\mathrm{a}}$ & $0.02^{\mathrm{a}}$ & $0.02^{\mathrm{a}}$ & 0.47 & 0.71 & \\
\hline Pyrrhosoma sp. & $0.02^{\mathrm{a}}$ & $0.02^{\mathrm{a}}$ & $0^{\mathrm{a}}$ & $0.02^{\mathrm{a}}$ & 0.38 & 0.77 & \\
\hline Argia sp. & $0^{\mathrm{a}}$ & $0^{\mathrm{a}}$ & $0.02^{\mathrm{a}}$ & $0^{\mathrm{a}}$ & 1 & 0.43 & \\
\hline Libellulidae & $0^{\mathrm{a}}$ & $0.02^{\mathrm{a}}$ & $0^{\mathrm{a}}$ & $0.02^{\mathrm{a}}$ & 0.62 & 0.62 & \\
\hline Pantala sp. & $0^{\mathrm{a}}$ & $0.02^{\mathrm{a}}$ & $0^{\mathrm{a}}$ & $0.00^{\mathrm{a}}$ & 1 & 0.43 & \\
\hline Unknown & $0^{\mathrm{a}}$ & $0^{\mathrm{a}}$ & $0^{\mathrm{a}}$ & $0.02^{\mathrm{a}}$ & 1 & 0.43 & \\
\hline ORTHOPTERA & $0.02^{\mathrm{a}}$ & $0.04^{\mathrm{a}}$ & $0.02^{\mathrm{a}}$ & $0^{\mathrm{a}}$ & 1 & 0.43 & \\
\hline Tettigonidae & $0.02^{\mathrm{a}}$ & $0.04^{\mathrm{a}}$ & $0.02^{\mathrm{a}}$ & $0^{\mathrm{a}}$ & 1 & 0.43 & \\
\hline Conocephalus longipennis & $0.02^{\mathrm{a}}$ & $0.04^{\mathrm{a}}$ & $0.02^{\mathrm{a}}$ & $0^{\mathrm{a}}$ & 1 & 0.43 & \\
\hline HYMENOPTERA & $0.11^{\mathrm{a}}$ & $0.65^{\mathrm{b}}$ & $0.35^{\mathrm{ab}}$ & $0.05^{\mathrm{a}}$ & 7.83 & 0 & 0.22 \\
\hline Formicidae & $0.11^{\mathrm{a}}$ & $0.64^{\mathrm{b}}$ & $0.35^{\mathrm{ab}}$ & $0.05^{\mathrm{a}}$ & 6.48 & 0.01 & 0.24 \\
\hline Odontoponera transversa & $0.09^{\mathrm{a}}$ & $0.64^{\mathrm{b}}$ & $0.35^{\mathrm{ab}}$ & $0.05^{\mathrm{a}}$ & 6.71 & 0.01 & 0.24 \\
\hline Solenopsis sp. & $0.02^{\mathrm{a}}$ & $0^{\mathrm{a}}$ & $0^{\mathrm{a}}$ & $0^{\mathrm{a}}$ & 1 & 0.43 & \\
\hline Syrphidae & $0^{\mathrm{a}}$ & $0.02^{\mathrm{a}}$ & $0^{\mathrm{a}}$ & $0^{\mathrm{a}}$ & 1 & 0.43 & \\
\hline Volucella sp. & $0^{\mathrm{a}}$ & $0.02^{\mathrm{a}}$ & $0^{\mathrm{a}}$ & $0^{\mathrm{a}}$ & 1 & 0.43 & \\
\hline MANTODEA & $0^{\mathrm{a}}$ & $0^{\mathrm{a}}$ & $0.02^{\mathrm{a}}$ & $0^{\mathrm{a}}$ & 1 & 0.43 & \\
\hline MANTIDAE & $0^{\mathrm{a}}$ & $0^{\mathrm{a}}$ & $0.02^{\mathrm{a}}$ & $0^{\mathrm{a}}$ & 1 & 0.43 & \\
\hline Unknown & $1.33^{\mathrm{b}}$ & $1.15^{\mathrm{b}}$ & $1.11^{\mathrm{b}}$ & $0.42^{\mathrm{a}}$ & 15.14 & 0 & 0.19 \\
\hline Total abundance $(\mathrm{N})$ & $14.6^{\mathrm{b}}$ & $12.7^{\mathrm{b}}$ & $12.2^{\mathrm{b}}$ & $4.6^{\mathrm{a}}$ & 17.00 & 0.00 & 0.74 \\
\hline
\end{tabular}

Note: Values within a row followed by the same letters were not significantly different at $\mathrm{P}<0.05$ according to Tukey's HSD test

Refugia as niche was capable of providing nectar and pollen as well as preys in form of herbivore or neutral insects. S. indicum was capable of providing nectar for parasitoid of rice stem borer (Zhu 2014a) and natural enemies (Coleoptera, Hymenoptera, Neuroptera, and Dictyoptera) (Mahmoud 2012). S. indicum was capable of increasing predator capability to prey on herbivores because its nectar and pollen can increase fitness and prey ability of this predator (Zhu 2014b). Feed-in form of nectar and pollen could prolong the longevity of predatory arthropods and parasitoid, and even synovigenic-type parasitoid urgently requires this feed in order to increase its egg production (Lu et al. 2014; Zhu 2014a). In addition, refugia existence on rice field embankments surrounding the rice could increase flora species diversity in rice fields. The higher the flora species is, the higher the fauna species diversity (arthropods) will be as flora inhabitant (Zhu 2014b). 
Table 4. Community characteristics of spiders inhabiting the rice plot surrounded by refugia, vegetables, untreated, and insecticide application for one planting season

\begin{tabular}{|c|c|c|c|c|c|}
\hline $\begin{array}{c}\text { Rice age } \\
\text { (days) }\end{array}$ & Community characteristics & $\begin{array}{c}\text { Rice- } \\
\text { refugia }\end{array}$ & $\begin{array}{c}\text { Rice- } \\
\text { vegetable }\end{array}$ & $\begin{array}{c}\text { Rice- } \\
\text { untreated }\end{array}$ & $\begin{array}{c}\text { Rice- } \\
\text { insecticide }\end{array}$ \\
\hline \multirow[t]{4}{*}{14} & Number of individual (individuals/net) & 0.4 & 0.2 & 0 & 1 \\
\hline & Diversity (H') & 0.69 & 0 & 0 & 0.67 \\
\hline & Dominance (D) & 0.5 & 1 & 0 & 0.6 \\
\hline & Evenness (E) & 1 & 0 & 0 & 0.97 \\
\hline \multirow[t]{4}{*}{21} & Number of individual (individuals/net) & 1.2 & 1.2 & 0.8 & 0.8 \\
\hline & Diversity (H') & 1.24 & 0.87 & 1.04 & 1.04 \\
\hline & Dominance (D) & 0.5 & 0.67 & 0.5 & 0.5 \\
\hline & Evenness (E) & 0.90 & 0.79 & 1.50 & 0.95 \\
\hline \multirow[t]{4}{*}{28} & Number of individual (individuals/net) & 2 & 1.8 & 2 & 0.8 \\
\hline & Diversity (H') & 1.64 & 1.68 & 1.33 & 1.04 \\
\hline & Dominance (D) & 0.3 & 0.33 & 0.4 & 0.5 \\
\hline & Evenness (E) & 0.92 & 0.94 & 0.96 & 0.95 \\
\hline \multirow[t]{4}{*}{35} & Number of individual (individuals/net) & 2.4 & 3 & 1 & 0.8 \\
\hline & Diversity (H') & 1.13 & 0.88 & 0.67 & 1.04 \\
\hline & Dominance (D) & 0.5 & 0.53 & 0.6 & 0.5 \\
\hline & Evenness (E) & 0.81 & 0.80 & 0.97 & 0.95 \\
\hline \multirow[t]{4}{*}{42} & Number of individual (individuals/net) & 2.2 & 2.6 & 2.4 & 1.6 \\
\hline & Diversity (H') & 1.04 & 1.31 & 0.82 & 1.04 \\
\hline & Dominance (D) & 0.45 & 0.38 & 0.67 & 0.5 \\
\hline & Evenness (E) & 0.94 & 0.94 & 0.75 & 0.95 \\
\hline \multirow[t]{4}{*}{49} & Number of individual (individuals/net) & 3.6 & 3 & 1.2 & 0.2 \\
\hline & Diversity (H') & 1.57 & 1.04 & 0 & 0 \\
\hline & Dominance (D) & 0.39 & 0.47 & 1 & 1 \\
\hline & Evenness (E) & 0.87 & 0.95 & 0 & 0 \\
\hline \multirow[t]{4}{*}{56} & Number of individual (individuals/net) & 2.6 & 2 & 1.2 & 0.4 \\
\hline & Diversity (H') & 1.18 & 0.64 & 0.69 & 0 \\
\hline & Dominance (D) & 0.62 & 0.8 & 0.5 & 1 \\
\hline & Evenness (E) & 0.73 & 0.58 & 1 & 0 \\
\hline \multirow[t]{4}{*}{63} & Number of individual (individuals/net) & 2.2 & 2.4 & 1 & 1.4 \\
\hline & Diversity (H') & 1.64 & 1.20 & 1.33 & 0.96 \\
\hline & Dominance (D) & 0.36 & 0.5 & 0.4 & 0.57 \\
\hline & Evenness (E) & 1.02 & 0.86 & 0.96 & 0.87 \\
\hline \multirow[t]{4}{*}{70} & Number of individual (individuals/net) & 3.2 & 2.2 & 1.2 & 1.2 \\
\hline & Diversity (H') & 1.72 & 1.47 & 0.64 & 1.56 \\
\hline & Dominance (D) & 0.31 & 0.36 & 0.67 & 0.33 \\
\hline & Evenness (E) & 0.88 & 0.91 & 0.92 & 0.97 \\
\hline \multirow[t]{4}{*}{77} & Number of individual (individuals/net) & 1.85 & 1.8 & 1.2 & 1 \\
\hline & Diversity (H') & 0.98 & 0.89 & 0.97 & 0.96 \\
\hline & Dominance (D) & 0.22 & 0.44 & 0.33 & 0.4 \\
\hline & Evenness (E) & 0.98 & 0.89 & 0.97 & 0.96 \\
\hline \multirow[t]{4}{*}{84} & Number of individual (individuals/net) & 3.2 & 2.8 & 1.4 & 2.85 \\
\hline & Diversity (H') & 2.06 & 1.77 & 0.80 & 1.58 \\
\hline & Dominance (D) & 0.31 & 0.36 & 0.71 & 0.49 \\
\hline & Evenness (E) & 0.90 & 0.91 & 0.72 & 0.81 \\
\hline \multirow[t]{4}{*}{ Total } & Number of individual (individuals/net) & 24.85 & 23 & 13.4 & 12.05 \\
\hline & Diversity (H') & 2.47 & 2.22 & 1.99 & 2.05 \\
\hline & Dominance (D) & 0.32 & 0.39 & 0.42 & 0.37 \\
\hline & Evenness (E) & 0.77 & 0.73 & 0.75 & 0.76 \\
\hline
\end{tabular}


Table 5. Community characteristics of predatory insects inhabiting the rice plot surrounded by refugia, vegetables, untreated, and insecticide application for one planting season

\begin{tabular}{|c|c|c|c|c|c|}
\hline $\begin{array}{c}\text { Rice age } \\
\text { (days) }\end{array}$ & Community characteristics & Rice-refugia & $\begin{array}{c}\text { Rice- } \\
\text { vegetable }\end{array}$ & $\begin{array}{c}\text { Rice- } \\
\text { untreated }\end{array}$ & $\begin{array}{c}\text { Rice- } \\
\text { insecticide }\end{array}$ \\
\hline \multirow[t]{4}{*}{14} & Number of individual (individuals/net) & 0.6 & 0.5 & 0.2 & 0.2 \\
\hline & Diversity (H') & 0.64 & 0 & 0 & 0 \\
\hline & Dominance (D) & 0.7 & 1 & 1 & 1 \\
\hline & Evenness (E) & 0.92 & 0 & 0 & 0 \\
\hline \multirow[t]{4}{*}{21} & Number of individual (individuals/net) & 1 & 0.6 & 1 & 0.2 \\
\hline & Diversity (H’) & 0.50 & 0.6 & 1.05 & 0 \\
\hline & Dominance (D) & 0.8 & 0.67 & 0.40 & 1 \\
\hline & Evenness (E) & 0.72 & 0.92 & 0.96 & 0 \\
\hline \multirow[t]{4}{*}{28} & Number of individual (individuals/net) & 0.8 & 1.6 & 0 & 0.4 \\
\hline & Diversity (H') & 0.56 & 0.74 & 0 & 0.69 \\
\hline & Dominance (D) & 0.81 & 0.67 & 0 & 1 \\
\hline & Evenness (E) & 0.75 & 0.75 & 0 & 0.50 \\
\hline \multirow[t]{4}{*}{35} & Number of individual (individuals/net) & 0.2 & 0.2 & 0.6 & 0 \\
\hline & Diversity (H') & 0 & 0 & 0 & 0 \\
\hline & Dominance (D) & 1 & 1 & 1 & 0 \\
\hline & Evenness (E) & 0 & 0 & 0 & 0 \\
\hline \multirow[t]{4}{*}{42} & Number of individual (individuals/net) & 0.8 & 1.6 & 0 & 0.2 \\
\hline & Diversity (H') & 0.56 & 1.26 & 0 & 0 \\
\hline & Dominance (D) & 0.75 & 0.38 & 0 & 1 \\
\hline & Evenness (E) & 0.81 & 0.91 & 0 & 0 \\
\hline \multirow[t]{4}{*}{49} & Number of individual (individuals/net) & 1.2 & 0.4 & 0 & 1 \\
\hline & Diversity (H') & 0 & 0 & 0 & 0 \\
\hline & Dominance (D) & 1 & 1 & 0 & 1 \\
\hline & Evenness (E) & 0 & 0 & 0 & 0 \\
\hline \multirow[t]{4}{*}{56} & Number of individual (individuals/net) & 2.2 & 2.4 & 3.6 & 0.8 \\
\hline & Diversity (H') & 1.34 & 0.57 & 1.38 & 0 \\
\hline & Dominance (D) & 0.36 & 0.83 & 0.56 & 1 \\
\hline & Evenness (E) & 0.97 & 0.52 & 0.77 & 0 \\
\hline \multirow[t]{4}{*}{63} & Number of individual (individuals/net) & 1.8 & 0.8 & 1 & 0 \\
\hline & Diversity (H') & 1.74 & 0.56 & 0 & 0 \\
\hline & Dominance (D) & 0.22 & 0.75 & 1 & 0 \\
\hline & Evenness (E) & 0.97 & 0.81 & 0 & 0 \\
\hline \multirow[t]{4}{*}{70} & Number of individual (individuals/net) & 1.4 & 0.6 & 1.4 & 0.6 \\
\hline & Diversity (H') & 0.96 & 0.64 & 0.6 & 0.64 \\
\hline & Dominance (D) & 0.57 & 0.67 & 0.71 & 0.67 \\
\hline & Evenness (E) & 0.87 & 0.92 & 0.86 & 0.92 \\
\hline \multirow[t]{4}{*}{77} & Number of individual (individuals/net) & 2.8 & 3 & 3.6 & 1 \\
\hline & Diversity (H') & 0.76 & 0 & 1.04 & 1.33 \\
\hline & Dominance (D) & 0.71 & 1 & 0.5 & 0.4 \\
\hline & Evenness (E) & 0.69 & 0 & 0.75 & 0.96 \\
\hline \multirow[t]{4}{*}{84} & Number of individual (individuals/net) & 1.8 & 1 & 0.8 & 0.2 \\
\hline & Diversity (H') & 1.89 & 1.33 & 1.39 & 0 \\
\hline & Dominance (D) & 0.22 & 0.4 & 0.25 & 1 \\
\hline & Evenness (E) & 0.97 & 0.96 & 1 & 0 \\
\hline \multirow[t]{4}{*}{ Total } & Number of individual (individuals/net) & 14.6 & 12.7 & 12.2 & 4.6 \\
\hline & Diversity (H') & 2.12 & 1.61 & 2.06 & 1.74 \\
\hline & Dominance (D) & 0.29 & 0.55 & 0.31 & 0.35 \\
\hline & Evenness (E) & 0.76 & 0.65 & 0.76 & 0.84 \\
\hline
\end{tabular}


This research showed that the abundance and species diversity of spider and predatory insects on the rice surrounded by refugia and vegetables were high and tended to have a higher community similarity for spiders and predatory insects than that of other plots. Vegetables $(C$. sativus, V. sinensis, L. acutangula, and M. charantia) have flowers that could provide nectar and pollen, but information related to arthropods associating with flowers on vegetables is not much. Lami et al. (2016) reported that predatory insects, such as ladybird beetles occurred during maize flowering in Northern and Central Italy. During this research period, the flowers of the four vegetables were frquently alight by the community of pollinating insects, spiders, predatory insects, and parasitoids of herbivores. The arthropods inhabiting those vegetables generally were M. sexmaculatus, $P$. fuscipes, Tetragnatha spp., and A. catenulata found on rice fields in this research.

The abundance and species diversity of spiders and predatory insects on the rice sprayed with synthetic insecticide were the lowest because the toxic from the insecticide could kill several arthropods species. This toxic kill not only herbivores, but also spider, predatory insects (Ngin et al. 2017), parasitoid (Svobodová et al. 2017), and pollinating insects (Sgolastra et al. 2016). The synthetic insecticide spraying can also slow down colonization of spider and predatory insects on those plots and can cause these natural enemies migration to other plots that are not sprayed with synthetic insecticide (Lopes et al. 2017).

Finally, the results of this study indicate that the most abundant predatory arthropods (spiders and predatory insects) were found in the rice surrounded by refugia flower and vegetables. The highest species diversity for spiders and predatory insects was also found on the rice surrounded by the refugia and vegetables. Therefore, the rice surrounded by the refugia and vegetables was the most appropriate for habitat and niche of predatory arthropods. Consequently, the refugia and vegetables should be planted along rice field embankments to conserve those useful arthropods.

\section{ACKNOWLEDGEMENTS}

This research was financially supported by Program of Professor Research Grant of Universitas Sriwijaya, Ministry of Research, Technology and Higher Education, Republic of Indonesia with the budget year of 2018, Contract Number: 0006/UN9/SK.LP2M.PT/2018, 5 June 2018 chaired by Siti Herlinda. We want to thank Program Beasiswa SDM IPTEK, Ministry of Research, Technology, and Higher Education that has given a scholarship for Tili Karenina.

\section{REFERENCES}

Baba YG, Kusumoto Y, Tanaka K. 2018. Effects of agricultural practices and fine-scale landscape factors on spiders and a pest insect in Japanese rice paddy ecosystems. BioControl 63 (2): 265-275.
Barrion A, Litsinger J. 1995. Riceland spiders of South and Southeast Asia. Entomology Division International Rice Research Institute, Philippines.

Cao MZ, Shen JL, Zhang JZ, Lu M, Liu XY, Zhou WJ. 2004. Monitoring of insecticide resistance and genetic analysis of Triazophos resistance in Chilo suppressalis (Lepidoptera: Pyralidae). Rice Sci 11: 297-304.

Desai SD, Swaminathan R, Desai VS. 2017. Effect of habitat manipulation on infestation of paddy leaf folder, Cnaphalocrocis medinalis (Guenee). Int J Curr Microbiol Appl Sci 6 (10): 1469-1477.

Dutton EM, Luo EY, Cembrowski AR, Shore JS, Frederickson ME. 2016. Three's a crowd: trade-offs between attracting pollinators and ant bodyguards with nectar rewards in turnera. J Am Nat 188 (1): 38-51.

Foti MC, Rosta M, Peri E, Park KC, Slimani T, Wratten SD, Colazza S. 2017. Chemical ecology meets conservation biological control: identifying plant volatiles as predictors of floral resource suitability for an egg parasitoid of stink bugs. J Pest Sci 90 (1): 299-310.

Ganai SA, Ahmad H, Sharma D, Sharma S, Khaliq N, Norboo T. 2017. Diversity of arthropod fauna associated with marigold (Tagetes erecta L.) in Jammu. J Entomol Zool Stud 5 (5): 1940-1943.

Hasan K, Pervin M, Manda F, Mala M. 2016. Habitat management: A key option to enhance natural enemies of crop pests. Universal J Plant Sci 4 (4): 50-57.

Heinrichs EA. 1994. Biology and management of rice insect. International Rice Research Institute. Philippines.

Herlinda S, Yudha S, Thalib R, Khodijah, Suwandi, Lakitan B, Verawaty M. 2018a. Species richness and abundance of spiders inhabiting rice in fresh swamps and tidal lowlands in South Sumatra, Indonesia. J ISSAAS 24 (1): 82-93.

Herlinda S, Apryanti H, Susilawati, Anggraini E. 2018b. Paddy rice spacing and its impact on community of insect pest in fresh swamp ecosystems. Indones J Entomol 15 (3): 151-165. [Indonesian].

Janzen DH. 2013. Differences in insect abundance and diversity between wetter and drier sites during a tropical dry season. Ecology 49 (1): 96110.

Jayakumar S, Sankari A. 2010. Spider population and their predatory efficiency in different rice establishment techniques in Aduthurai, Tamil Nadu. J Biopesticides 3 (1): 20-27.

Kalshoven LG. 1981. The pest of crops in Indonesia. Van Hoeve. Jakarta.

Kamarudin N, Arshad O. 2016. Diversity of insect natural enemies of the bagworm, Pteroma pendula (Lepidoptera: Psychidae) in relation to the oil palm environment, in Perak, Malaysia. J Oil Palm Res 28 (3): 296-307.

Lakitan B, Alberto A, Lindiana L, Kartika K, Herlinda S, Kurnianingsih, A. 2018a. The benefits of biochar on rice growth and yield in tropical riparian wetland, South Sumatra, Indonesia. Chiang Mai University J Nat Sci 17 (2): 111-126.

Lakitan B, Hadi B, Herlinda S, Siaga E, Widuri LI, Kartika K, Lindiana L, Yunindyawati Y, Meihana M. 2018b. Recognizing farmers' practices and constraints for intensifying rice production at riparian wetlands in Indonesia. NJAS-Wageningen J Life Sci 85: 10-20.

Lami F, Masetti A, Neri U, Lener M, Staiano G, Arpai S, Burgio G. 2016. Diversity of Coccinellidae in ecological compensation areas of Italy and overlap with maize pollen shedding period. Bull Insect 69 (1): 4957.

Lopes SF, Ramos MB, Almeida GR. 2017. The role of mountains as refugia for biodiversity in Brazilian Caatinga: conservationist implications. Trop Conserv Sci 10: 1-12.

Lu ZX, Zhu PY, Gurr GM, Zheng XS, Read DM, Heong KL, Yang YJ, $\mathrm{Xu}$ HX. 2014. Mechanisms for flowering plants to benefit arthropod natural enemies of insect pests: Prospects for enhanced use in agriculture. Insect Sci 21: 1-12.

Mahmoud MF. 2012. Insects associated with sesame (Sesamun indicum L.) and the impact of insect pollinators on crop production. Pestic Phytomed 27 (2): 117-129.

Masika FB, Masanza M, Aluana G, Barrigossi JAF, Kizito EB. 2017. Abundance, distribution and effects of temperature and humidity on arthropod fauna in different rice ecosystems in Uganda. J Entomol Zool Stud 5 (5): 964-973.

Mcalpine JF, Peterson BV, Shewel GE, Teskey HJ, Vockeroth JR, Wood DM. 1987. Manual of neartic Diptera. Agriculture Canada. Research Branch.

Mccabe E, Loeb G, Grab H. 2017. Responses of crop pests and natural enemies to wildflower borders depends on functional group. Insects 8 (3): $1-8$. 
Ngin C, Suon S, Tanaka T, Yamauchi A, Kawakita K, Chiba S. 2017. Impact of insecticide applications on arthropod predators and plan feeders in Cambodian rice fields. Phytobiomes J 1: 128-137.

Saleh A, Siregar AZ. 2017. Impact of natural enemies to leaf eating caterpillar population on oil palm in north Sumatra, Indonesia. Int J Sci Technol Res 6 (8): 189-192.

Safitri A, Herlinda S, Setiawan A. 2018. Entomopathogenic fungi of soils of freshwater swamps, tidal lowlands, peatlands, and highlands of South Sumatra, Indonesia. Biodiversitas 19: 2365-2373.

Sgolastra F, Fisogni A, Quaranta M, Bogo G, Bortolotti L, Galloni M. 2016. Temporal activity patterns in a flower visitor community of Dictamnus albus in relation to some biotic and abiotic factors. Bull Insectology 69 (2): 291-300.

Suwandi, Ammar M, Irsan C. 2012. Application of extract compost increased yield and suppressed the diseases of ratoon rice crop in tidal swamp of Banyuasin Regency. Jurnal Lahan Suboptimal 1 (2): 116 122. [Indonesian].
Svobodová Z, Skokova HO, Holec J, Holec M, Bohac M, Bohac J, Jursic M, Soukup J, Sehnal F. 2017. Split application of glyphosate in herbicide-tolerant maize provides efficient weed control and favors beneficial epigeic arthropods. Agric Ecosyst Environ 251: 171-179.

Xu HX, Yang YJ, Lu YH, Zheng XS, Tian JE, Lai FX, Fu Q, Lu ZX. 2017. Sustainable management of rice insect pests by non-chemicalinsecticide technologies in China. Rice Sci 24 (2): 61-72.

Zhu P, Lu Z, Heong K, Chen G, Zheng X, Xu H, Yang Y, Nicol HL, Gurr GM. 2014a. Selection of nectar plants for use in ecological engineering to promote biological control of rice pests by the predatory bug, Cyrtorhinus lividipennis, (Heteroptera: Miridae). Plos One 9 (9): 1-12.

Zhu P, Wang G, Zheng X, Tian J, Lu Z, Heong KK, Xu H, Chen G, Yang Y, Gurr GM. 2014b. Selective enhancement of parasitoids of rice Lepidoptera pests by sesame (Sesamum indicum) flowers. BioControl 60: 157-167. 